\title{
Automated Segmentation of 3D US Prostate Images Using Statistical Texture-Based Matching Method
}

\author{
Yiqiang Zhan ${ }^{1,2,3}$ and Dinggang Shen ${ }^{1,2}$ \\ ${ }^{1}$ Sect. of Biomedical Image Analysis, Dept. of Radiology, \\ University of Pennsylvania, Philadelphia, PA \\ ${ }^{2}$ Center for Computer-Iyntegrated Surgical Systems and Technology, \\ Johns Hopkins University, Baltimore, MD \\ ${ }^{3}$ Dept. of Computer Science, Johns Hopkins University, Baltimore, MD \\ \{yzhan, dgshen\} @rad. upenn. edu
}

\begin{abstract}
A novel statistical shape model is presented for automatic and accurate segmentation of prostate boundary from 3D ultrasound (US) images, using a hierarchical texture-based matching method. This method uses three steps. First, Gabor filter banks are used to capture rotation-invariant texture features at different scales and orientations. Second, different levels of texture features are integrated by a kernel support vector machine (KSVM) to optimally differentiate the prostate from surrounding tissues. Third, a statistical shape model is hierarchically deformed to the prostate boundary by robust texture and shape matching. Experimental results test the performance of the proposed method in segmenting 3D US prostate images.
\end{abstract}

\section{Introduction}

Prostate cancer is the second-leading cause of cancer deaths in American men. As transrectal ultrasound (TRUS) images have been widely used for the diagnosis and treatment of prostate cancer, the accurate segmentation of the prostate from TRUS images plays an important role in many applications. A number of automatic or semiautomatic segmentation methods have been proposed, to date. Richard and Keen [2] presented a texture-based segmentation method that uses four texture energies to classify pixels into prostate or non-prostate tissues. Ghanei et.al. [1] and $\mathrm{Hu}$ et.al. [8] designed a 3D discrete deformable model to semi-automatically outline the prostate boundaries. Shao et.al.[3] proposed a level set method to detect the prostate in the 3D TRUS images.

We previously proposed a statistical shape model to segment the prostate from $2 \mathrm{D}$ TRUS images, using hierarchical matching of the texture features calculated by $2 \mathrm{D}$ Gabor filters [4]. This method is further expanded in this paper to permit the automated segmentation of 3D TRUS prostate images. The expanded method has two major novelties. First, two banks of 2D Gabor filters placed at the orthogonal planes are employed to extract 3D texture features in a multi-scales and multi-orientations 
fashion. Compared to the direct use of 3D Gabor filters for extracting 3D texture features, the use of two banks of 2D Gabor filters saves the computation time, and still preserves the significant 3D texture features. Second, the prostate is differentiated from the surrounding tissues via a kernel support vector machine (KSVM) that has been trained by Gabor texture features obtained from a group of TRUS image samples. The joint use of these two novel techniques facilitates the deformable model to find the desired prostate boundaries in the 3D TRUS images.

\section{Methods}

Our deformable shape model uses both shape statistical information and image texture information to segment the prostate boundary. The shape statistics is collected from a set of hand-labeled samples, and it is used to constrain the shape of the model during the deformable segmentation stages. The image texture is represented by Gabor features, and used to distinguish the boundary between the prostate and the nonprostate regions. Due to page limits, we will not discuss the procedures for collecting shape statistics and constraining the model's shape during the progress of deformation. The details of these procedures can be found in [6]. This section will mainly focus on differentiating the prostate from surrounding tissues by using the texture analysis method.

The goal of texture analysis is to classify tissue textures in the TRUS images, in order to distinguish prostate and non-prostate regions. The correct classification of tissue textures is highly related to (i) the representation of texture, and (ii) the performance of the classifier. To achieve the desired tissue classification, we need to collect the texture features that are discriminative for the prostate and the surrounding tissues. Also, we need to employ a powerful classifier to distinguish the prostate tissues from the surrounding tissues, as the distribution of some texture features of the prostate may overlap with those of the surrounding tissues. A simple linear classifier may be not effective, since the corresponding texture features of the prostate vary greatly across individuals. Also, a single classifier may be insufficient to correctly label all prostate tissues in the whole volumetric image, due to the high diversity of the texture features along the prostate boundary.

In this study, Gabor filters are used to extract texture features around each voxel at multiple scales and orientations, and KSVM is used to measure the probability of voxels belonging to the prostate, based on their Gabor features. The input to the KSVM classifier is Gabor features extracted at multi-scales and multi-orientations, and its output measures the probability of a voxel belonging to the prostate. By using this texture analysis method, the prostate is optimally differentiated from surrounding tissues, thereby facilitating the deformable model to find the desired prostate boundary in 3D TRUS images. The details of this texture analysis method are provided next. 


\subsection{Gabor Filters for Extracting Texture Features}

The Gabor filter bank is capable of extracting robust and rich image features for texture representation and classification [7], because the structure of the Gabor filter bank's frequency spectrum works both in a multi-scale and multi-orientation fashion (c.f. Fig 1). Therefore, different levels of texture features can be extracted. Also, as a complex filter, each Gabor filter can be classified into real part and imaginary part, which can be used as a smooth filter and an edge detection filter, respectively, by appropriately selecting the parameters in the Gabor filter [4]. Thereby, the noises in the TRUS images can be removed, and the edge direction as well as the edge strength can be extracted completely.

To save computation time and also to best approximate the complete set of 3D Gabor features, two banks of 2D Gabor filters located at the orthogonal planes are used. As shown in Fig 1, the two selected filter banks lie on the two planes, i.e., on the axial and coronal planes, which are respectively orthogonal to or parallel with the direction of ultrasound probe. With this design of 2D Gabor filter banks, the extracted features are sufficient to characterize 3D prostate textures in our study, and importantly there is a dramatic saving in the time used to compute the Gabor features.

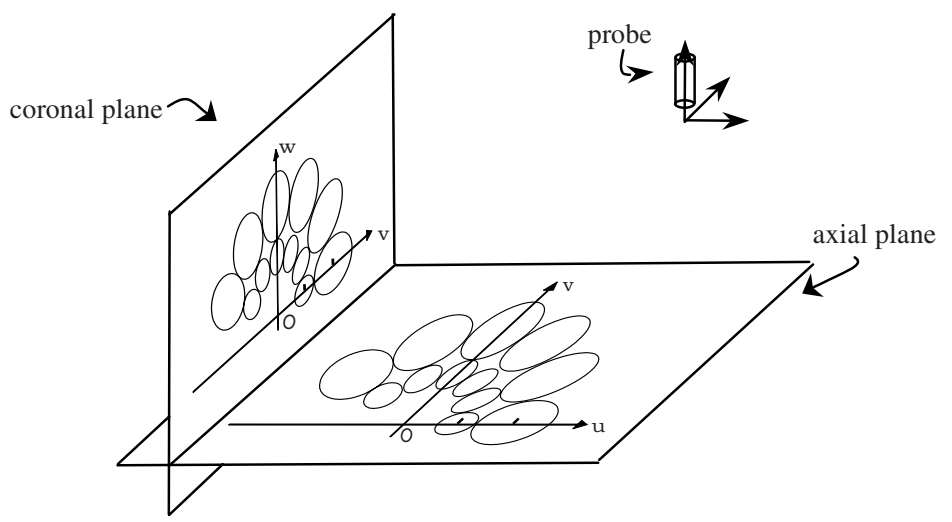

Fig. 1. The frequency spectrums of two banks of Gabor filters located at the two orthogonal planes. The ellipse contour denotes the half-peak magnitude of the filter responses. The axes $u$, $v$ and $w$, respectively, denote three orthogonal directions in the 3D spatial frequency space. For display purposes, the two Cartesian coordinates are shifted, although they actually have the same origin $O$. The small figure in the top-right corner indicates the positions of the axial and the coronal planes with respect to the direction of the ultrasound probe.,

Two banks of Gabor filters at two orthogonal planes are represented as,

$$
\begin{array}{r}
g_{s, k}(x, y)=a^{s} g\left(a^{s}(x \cos (k \psi)+y \sin (k \psi)), a^{s}(-x \sin (k \psi)+y \cos (k \psi))\right) \\
h_{s, k}(y, z)=a^{s} h\left(a^{s}(y \cos (k \psi)+z \sin (k \psi)), a^{s}(-y \sin (k \psi)+z \cos (k \psi))\right) \\
(s=0, \cdots, S-1 ; k=0, \cdots, K-1)
\end{array}
$$


with the respective mother functions,

$$
\begin{aligned}
& g(x, y)=\left(\frac{1}{2 \pi \sigma_{x} \sigma_{y}}\right) \exp \left[-\frac{1}{2}\left(\frac{x^{2}}{\sigma_{x}^{2}}+\frac{y^{2}}{\sigma_{y}^{2}}\right)+2 \pi j W x\right] \\
& h(y, z)=\left(\frac{1}{2 \pi \sigma_{y} \sigma_{z}}\right) \exp \left[-\frac{1}{2}\left(\frac{y^{2}}{\sigma_{y}^{2}}+\frac{z^{2}}{\sigma_{z}^{2}}\right)+2 \pi j W y\right]
\end{aligned}
$$

Here, the total number of orientations and scales are $K$ and $S$, respectively (i.e., $K=6$ and $S=2$ in this study). The basic rotation and scale factors are $\psi=\pi / K$ and $a=(U h / U l)^{\frac{1}{S-1}}$, respectively. $U h$ and $U l$ are the parameters that determine the frequency range of the Gabor filter bank, and $W$ is a shifting parameter used in the frequency domain. Fig 1 shows the frequency spectrums of Gabor filters.

By using the Gabor filters designed above, we can obtain four sets of features for each voxel $\vec{v}$, corresponding to the real and the imaginary parts of the two filter banks, as given next.

$$
\begin{aligned}
\left\{\widehat{G}^{\text {real }}{ }_{s, k}(\vec{v})\right\},\left\{\widehat{G}^{\text {img }}(\vec{v})\right\}, & \left\{H_{s, k}^{\text {real }}(\vec{v})\right\},\left\{H_{s, k}^{\text {img }}(\vec{v})\right\} \\
& (s=0, \cdots, S-1 ; k=0, \cdots, K-1)
\end{aligned}
$$

Notably, since the features extracted by the Gabor filter bank at the axial plane are not invariant to the rotation of the ultrasound probe, these features are reconstructed to be rotation-invariant features as $\widehat{G}$ in equation (3), by a procedure detailed in [4]. All of these Gabor features can be compiled into a feature vector $\vec{F}(\vec{v})$, and used as texture features for the voxel $\vec{v}$. As the real part of the Gabor filter acts as a smooth filter and is insensitive to orientations, in applications the feature vector $\vec{F}(\vec{v})$ includes only two real Gabor features per scale $s$, i.e., $\widehat{G}^{\text {real }}{ }_{s, 0}(\vec{v})$ and $H^{\text {real }}(\vec{v})$, in order to reduce the redundancy in $\vec{F}(\vec{v})$.

\subsection{KSVM for Classifying Tissues}

In this section, a pattern classification method is applied to softly classify each voxel as prostate or non-prostate tissue, by using the Gabor feature vector defined above. Since the Gabor features of TRUS prostate images vary greatly across the individuals and their distribution is highly overlapped between prostate and non-prostate regions. Therefore, only non-linear classifiers can determine the probability of a voxel belonging to the prostate. KSVM is selected as a tissue classifier in this study, since it is a well-known and powerful non-linear classifier and has been extensively used in the area of pattern recognition.

Given $m$ labeled samples, $\left\{\left(\vec{x}_{i}, y_{i}\right) \mid \vec{x}_{i} \in R^{n}, y_{i} \in\{-1,+1\}, i=1 \cdots m\right\}$, KSVM can create a hyperplane that separates the positive samples from the negative ones [5]. This is related to the two important techniques employed in KSVM. First, KSVM addresses the non-linear separation problem by mapping samples $\left\{\vec{x}_{i}\right\}$ to a higher 
dimensional space via the "kernel trick" [5]. Second, KSVM selects "support vectors" to classify the samples in an effective manner. The following three targeted techniques make our method particularly effective and robust.

First, rather than outputting a binary value as a label for the input voxel, the KSVM classifier is modified to output a real value, which denotes the signed distance from the feature vector of a voxel to the decision hyperplane. This value is further mapped to $[0,1]$ by a sigmoid function, and used as the probability of the voxel belonging to the prostate.

Second, we use a set of KSVMs rather than a single KSVM to classify the voxels, due to the high diversity of the texture features along the prostate boundary. In the training stage, we divide the prostate model into a set of non-overlapping subsurfaces, and assign each subsurface a KSVM classifier. Each KSVM is trained by the handlabeled prostate and non-prostate samples that are close to the subsurface. In the application stage, the trained KSVM classifiers are used to measure the probabilities of voxels belonging to the prostate.

Third, as the prostate voxels and the non-prostate voxels come to have very similar texture features if they are contiguous to the prostate boundary, we select only the voxel samples that are a little bit far from the manually outlined prostate boundary, to avoid including the very similar samples into both classes. This caution allows better training of KSVMs. For example, as shown in Fig 2, the voxels outside of the prostate boundary are selected as negative training samples, i.e., labeled as -1; while the voxels inside of the prostate boundary are selected as positive training samples, i.e., labeled as +1 .

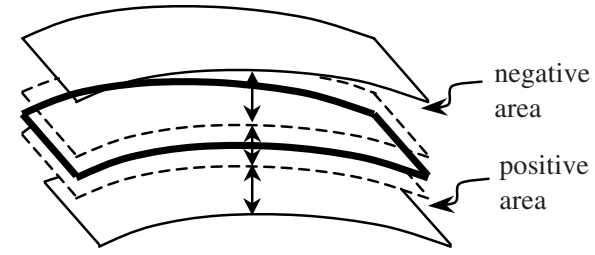

Fig. 2. Schematic explanation of how appropriate samples for training a KSVM are selected. The thick, solid surface denotes the prostate boundary. The region between two dashed surfaces denotes the area from which the voxel samples will not be selected. The region between the upper dashed and the upper thin surfaces denotes the area of negative samples, while the region between the lower dashed and the lower thin surfaces denotes the area of positive samples.

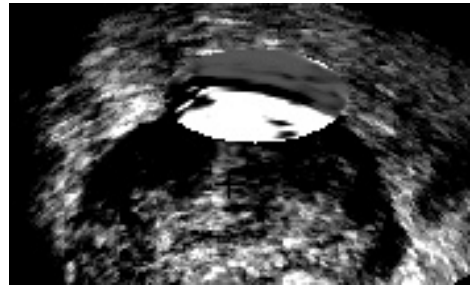

Fig. 3. The results of tissue classification using a KSVM classifier, shown only in an ellipsoidal region for comparison. For display purposes, the tissue-labeling results, which originally ranged from 0 to 1 , has been mapped to $0 \sim 255$.

In the application stage, each subsurface uses its assigned KSVM classifier to classify the voxels in the neighborhood. For example, if the voxel $\vec{v}$ is in the neighborhood of the $j$-th subsurface of the prostate model, the probability of this voxel belonging to prostate is mathematically defined as: 


$$
T(\vec{v} ; j)=s\left(\sum_{i=1}^{m^{j}} \alpha_{i}^{j} y_{i}^{j} K\left(\vec{F}_{i}^{j}, \vec{F}(\vec{v})\right)+b^{j}\right)
$$

where $s(x)=1 /\left(1+e^{-x}\right) \cdot m^{j}$ is the sample number of the $j$ 'th subsuface. $K(\cdot, \cdot)$ denotes a Gaussian kernel function, which maps the samples to a higher dimension. $\alpha_{i}^{j}$ and $b^{j}$ are parameters that are determined by the optimal hyperplane algorithm [5]. $\vec{F}_{i}^{j}$ is the Gabor feature vector of the $i$-th training sample of the $j$-th subsuface, with the assigned label $y_{i}^{j} \in\{-1,+1\} . \vec{F}(\vec{v})$ is the Gabor feature vector of a voxel $\vec{v}$ under study. As shown in Fig 3, the prostate is differentiated from the surrounding tissues, using the KSVM-based tissue classification.

\subsection{Energy Function}

Energy function is used to evaluate the matching degree of the deformable model with the prostate boundary in the TRUS images. In our case, two energy terms are defined on each vertex of the surface model, i.e., the external energy and the internal energy. The internal energy is defined by the geometric attribute vectors that reflect the geometric properties of the underlying structure from a local to a global scale, and is used to preserve the geometric regulation of the model during deformation [6]. The external energy is defined by tissue classification results and is used to drive the deformable model to the prostate boundary. In the following, we will focus on formulating the external energy.

Since the KSVM-based tissue classification method differentiates the prostate from the surrounding tissues, the external energy on each vertex $\vec{P}_{i}$ can be defined by analyzing the tissue classification results in its neighborhood, $N($.). If the vertex under study is precisely localized on the prostate boundary, then in the spherical neighborhood of this vertex, (i) the number of the voxels labeled as prostate tissues should be close to the number of the voxels labeled as the non-prostate tissues, (ii) the prostate voxels and non-prostate voxels should be symmetrically distributed around the studied vertex, such as the centers of the prostate and the non-prostate regions should be similarly distant from the studied vertex (Fig 4).

Accordingly, the external energy can be mathematically defined for the vertex $\vec{P}_{i}$ that belongs to the $j$-th subsurface as follows.

$$
\begin{gathered}
E^{\mathrm{Ext}}\left(\vec{P}_{i}\right)=w_{\text {Sum }} T_{\text {Sum }}\left(\vec{P}_{i}\right)+w_{\text {Dist }} T_{\text {Dist }}\left(\vec{P}_{i}\right) \\
T_{\text {Sum }}\left(\vec{P}_{i}\right)=\left(\left(\sum_{\forall \vec{v} \in N\left(\vec{P}_{i}\right)} T(\vec{v} ; j) / \sum_{\forall \vec{v} \in N\left(\vec{P}_{i}\right)} 1\right)-0.5\right)^{2}, T_{\text {Dist }}\left(\vec{P}_{i}\right)=\left(d\left(\vec{C}_{\mathrm{P}}, \vec{P}_{i}\right)-d\left(\vec{C}_{\mathrm{NP}}, \vec{P}_{i}\right)\right)^{2} / R^{2}
\end{gathered}
$$


Here, $N($.$) denotes the spherical neighborhood, with the radius R$, around the vertex $\vec{P}_{i} . d(.,$.$) denotes the 3D Euclidean distance of two points. \vec{C}_{\mathbf{P}}$ and $\vec{C}_{\mathrm{NP}}$ denote the centers of the prostate and the non-prostate regions, respectively.

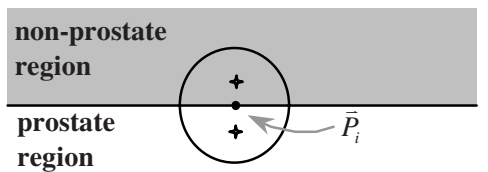

Fig. 4. The distribution of the prostate and non-prostate regions in the case of the studied vertex $\vec{P}_{i}$ precisely locating on the prostate boundary. The gray region denotes the non-prostate region, with output values from KSVM less than 0.5. The white region denotes the prostate region, with output values larger than 0.5 . The black point denotes the vertex on the prostate boundary. The circle denotes the spherical neighborhood of the studied vertex. The lower and upper crosses denote the centers of prostate region and non-prostate region, respectively.

Table 1. Comparison of the automated and the hand-labeled segmentation results.

\begin{tabular}{lccc}
\hline & $\begin{array}{c}\text { Avg. Distance } \\
\text { (Voxels) }\end{array}$ & $\begin{array}{c}\text { Overlap Volume } \\
\text { Error (\%) }\end{array}$ & $\begin{array}{c}\text { Total Volume } \\
\text { Error (\%) }\end{array}$ \\
\hline Individual 1 & 0.76 & 2.6 & 0.2 \\
Individual 2 & 0.88 & 3.7 & 1.7 \\
Individual 3 & 0.79 & 5.5 & 2.6
\end{tabular}
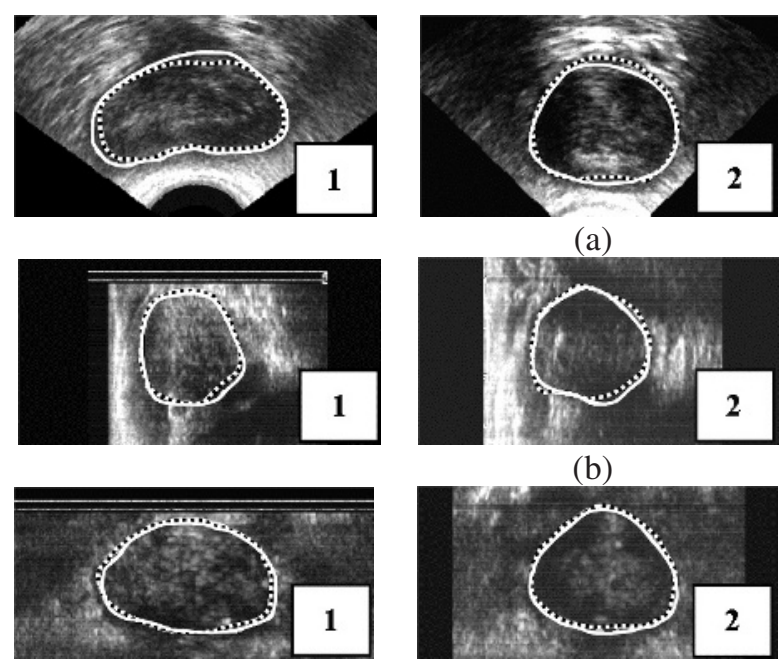

(a)

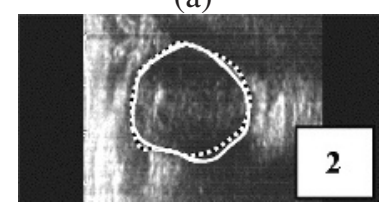

(b)

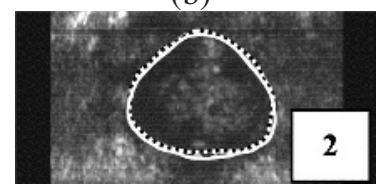

(c)
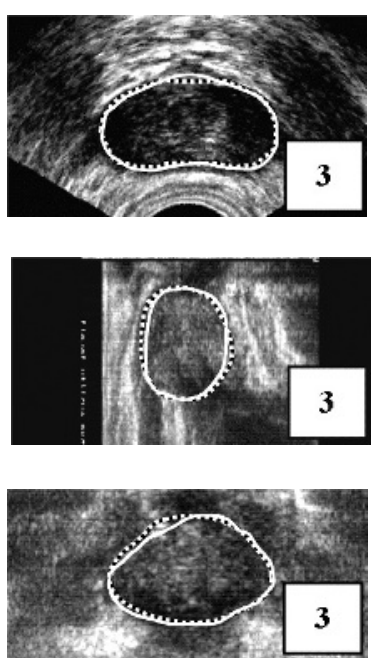

3

Fig. 5. Visual comparison of automated and hand-labeled segmentation results of three selected individuals. The solid contours are the hand-labeled results, and the dashed ones are the automated segmentation results. (a) Axial view, (b) Sagittal view, (c) Coronal view. 


\section{Experiments}

The performance of our approach is tested by comparing the automated prostate segmentation results with the hand-labeled results on a group of 3D TRUS images. The accuracy of the automated segmentations can be visually observed by the results in Fig 5. Moreover, Table 1 gives a quantitative evaluation of this comparison. The size of $3 \mathrm{D}$ images is $256 \times 256 \times 176$, with the spatial resolution $0.306 \mathrm{~mm}$. From both visual results and quantitative analysis, we can conclude that our automated segmentation method is able to segment the prostate from noisy TRUS images of a small group of individuals. We are planning a large-scale validation study of our method.

\section{Conclusion}

In this paper, a novel approach for automatically segmenting prostates from 3D TRUS images has been proposed. Gabor filters are used to represent the tissue textures in the TRUS prostate images, and the KSVM is used to measure the probability of a voxel belonging to the prostate, in order to differentiate the prostate from surrounding tissues. The use of this texture analysis method facilitates our surface model to find the desired prostate boundaries in the 3D TRUS images.

Our future work will focus on improving the efficiency of the approach in the following two ways. First, additional hierarchical strategies will be studied and employed in our approach. For example, the Gabor feature vector can be dissembled into sub-vectors, according to the scales of the Gabor filters, thereby the probability of a voxel belonging to the prostate can be hierarchically obtained and further used to drive the model's deformation at a hierarchical fashion. Second, because the calculation of Gabor features occupies the majority of computation time in our approach, the efficient extraction of Gabor features will be studied.

\section{References}

1. A. Ghanei, H. Soltanian-Zadeh, A. Ratkesicz and F. Yin, "A three-dimensional deformable model for segmentation of human prostate from ultrasound image", Medical Physics, Vol. 28, pp. 2147-2153, 2001.

2. W.D. Richard and C.G. Keen, "Automated Texture-based Segmentation of Ultrasound Images of the Prostate", Computerized Medical Imaging and Graphics, Vol. 20, pp. 131$140,1996$.

3. F. Shao, K.V. Ling and W.S. Ng, "3D Prostate Surface Detection from Ultrasound Images Based on Level Set Method”, MICCAI 2002, pp. 389-396, 2002.

4. D. Shen, Y. Zhan and C. Davatzikos, "Segmentation Prostate Boundaries from Ultrasound Images Using Statistical Shape Model”, IEEE Trans. on Med. Img., Vol.22, pp. 539-551, Apr.2003.

5. C.J.C. Burges, "A Tutorial on Support Vector Machines for Pattern Recognition", Data Mining and Knowledge Discovery, Vol. 2, pp. 121-167, 1998. 
6. D.Shen, E.H.Herskovits and C.Davatzikos, "An adaptive-focus statistical shape model for segmentation and shape modeling of 3D brain structures", IEEE Trans. on Med. Img., Vol. 20, pp. 257-270, 2001.

7. B.S. Manjunath and W.Y. Ma, "Texture Features for Browsing and Retrieval of Image Data”, IEEE Trans. on Pattern Anal. Mach. Intell., Vol. 18, pp. 837-842, 1996.

8. N. Hu, D. Downey, A. Fenster, and H. Ladak, "Prostate surface segmentation from 3D ultrasound images", IEEE International Symposium on Biomedical Imaging, pp. 613-616, Washington, D. C., 2002. 